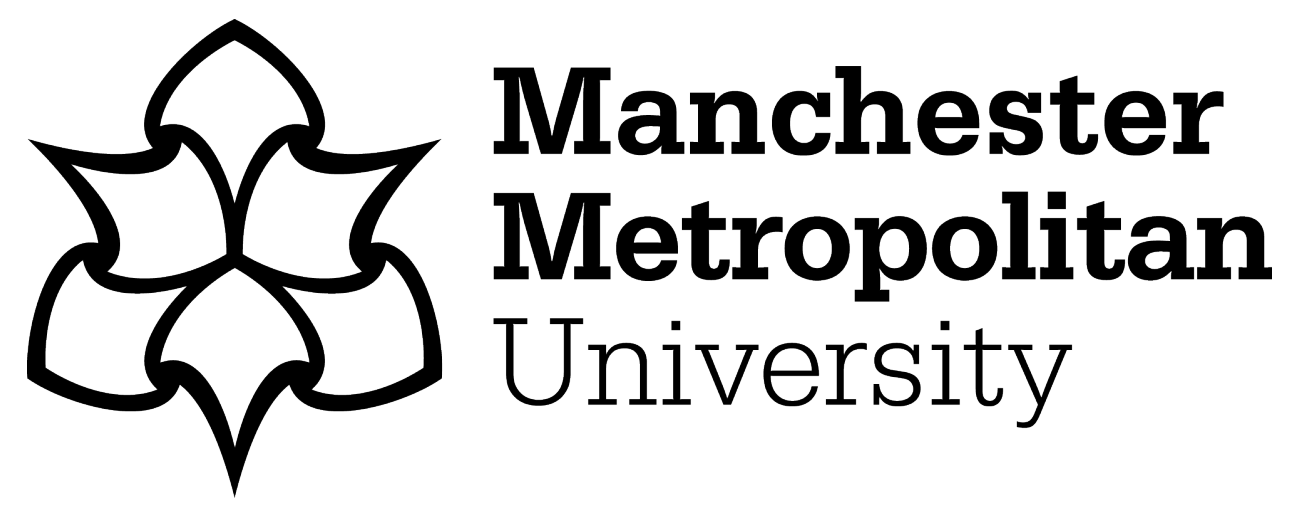

Lynch, Stephen (2018) Dynamical Systems with Applications using Python. Birkhäuser. ISBN 9783319781457

Downloaded from: https://e-space.mmu.ac.uk/622016/

Publisher: Birkhäuser

DOI: https://doi.org/10.1007/978-3-319-78145-7

Please cite the published version 


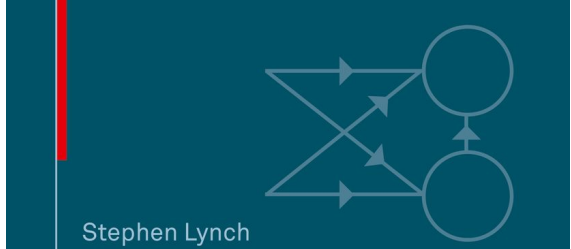

Stephen Lynch

\section{Dynamical Systems with Applications using Python}

קै Birkhäuser

1st ed. 2018, XVI, 665 p. 277 illus., 118 illus. in color.

\section{Printed book}

Hardcover

$79,99 €|£ 69.99| \$ 89.99$

${ }^{[1]} 85,59 €(D)|87,99 €(A)| C H F$

94,50

eBook

$67,82 €|£ 55.99| \$ 69.99$

${ }^{[2]} 67,82 €(D)|67,82 €(A)| C H F$

75,50

Available from your library or

springer.com/shop

MyCopy [3]

Printed eBook for just

$€$ | \$ 24.99

springer.com/mycopy
Stephen Lynch

\section{Dynamical Systems with Applications using Python}

- Designed for a broad audience of students in applied mathematics, physics, and engineering

- Represents dynamical systems with popular Python libraries like sympy, numpy, and matplotlib

- Explores a variety of advanced topics in dynamical systems, like neural networks, fractals, and nonlinear optics, at an undergraduate level

This textbook provides a broad introduction to continuous and discrete dynamical systems. With its hands-on approach, the text leads the reader from basic theory to recently published research material in nonlinear ordinary differential equations, nonlinear optics, multifractals, neural networks, and binary oscillator computing. Dynamical Systems with Applications Using Python takes advantage of Python's extensive visualization, simulation, and algorithmic tools to study those topics in nonlinear dynamical systems through numerical algorithms and generated diagrams. After a tutorial introduction to Python, the first part of the book deals with continuous systems using differential equations, including both ordinary and delay differential equations. The second part of the book deals with discrete dynamical systems and progresses to the study of both continuous and discrete systems in contexts like chaos control and synchronization, neural networks, and binary oscillator computing. These later sections are useful reference material for undergraduate student projects. The book is rounded off with example coursework to challenge students' programming abilities and Python-based exam questions. This book will appeal to advanced undergraduate and graduate students, applied mathematicians, engineers, and researchers in a range of disciplines, such as biology, chemistry, computing, economics, and physics.
Order online at springer.com / or for the Americas call (toll free) 1-800-SPRINGER /

or email us at: customerservice@springernature.com. / For outside the Americas call +49 (0) 6221-345-4301 / or email us at: customerservice@springernature.com.

The first $€$ price and the $£$ and \$ price are net prices, subject to local VAT. Prices indicated with [1] include VAT for books; the $€(D)$ includes $7 \%$ for Germany, the $€(A)$ includes $10 \%$ for Austria. Prices indicated with [2] include VAT for electronic products; 19\% for Germany, 20\% for Austria. All prices exclusive of carriage charges. Prices and other details are subject to change without notice. All errors and omissions excepted. [3] No discount for MyCopy. 
Q Birkhäuser 
Stephen Lynch

Dynamical Systems with

Applications using Python

Birkhäuser 


\author{
Stephen Lynch \\ Manchester Metropolitan University \\ Manchester, UK
}

ISBN 978-3-319-78144-0

ISBN 978-3-319-78145-7 (eBook)

https://doi.org/10.1007/978-3-319-78145-7

Library of Congress Control Number: 2018952351

Mathematics Subject Classification (2010): 00A05, 00A69, 37-01, 34-01, 34Cxx, 34Dxx, 34H10, 34K18, 37-04, 37Nxx, 68U10, 78A60, 92B20

(C) Springer International Publishing AG, part of Springer Nature 2018

This work is subject to copyright. All rights are reserved by the Publisher, whether the whole or part of the material is concerned, specifically the rights of translation, reprinting, reuse of illustrations, recitation, broadcasting, reproduction on microfilms or in any other physical way, and transmission or information storage and retrieval, electronic adaptation, computer software, or by similar or dissimilar methodology now known or hereafter developed.

The use of general descriptive names, registered names, trademarks, service marks, etc. in this publication does not imply, even in the absence of a specific statement, that such names are exempt from the relevant protective laws and regulations and therefore free for general use.

The publisher, the authors and the editors are safe to assume that the advice and information in this book are believed to be true and accurate at the date of publication. Neither the publisher nor the authors or the editors give a warranty, express or implied, with respect to the material contained herein or for any errors or omissions that may have been made. The publisher remains neutral with regard to jurisdictional claims in published maps and institutional affiliations.

This book is published under the imprint Birkhäuser, www.birkhauser-science.com by the registered company Springer Nature Switzerland AG

The registered company address is: Gewerbestrasse 11, 6330 Cham, Switzerland 


\section{Preface}

This book provides an introduction to the theory of dynamical systems with the aid of Python. It is written for both senior undergraduates and graduate students. Chapter 1 provides a tutorial introduction to Python-new users should go through this chapter carefully while those moderately familiar and experienced users will find this chapter a useful source of reference. The first part of the book deals with continuous systems using differential equations, including both ordinary and delay differential equations (Chapters 2-12), the second part is devoted to the study of discrete systems (Chapters 13-17), and Chapters 18-21 deal with both continuous and discrete systems. Chapter 22 gives examples of coursework and also lists three Python-based examinations to be sat in a computer laboratory with access to Python. Chapter 23 lists answers to all of the exercises given in the book. It should be pointed out that dynamical systems theory is not limited to these topics but also encompasses partial differential equations, integral and integro-differential equations, and stochastic systems, for instance. References [1-6] given at the end of the Preface provide more information for the interested reader. The author has gone for breadth of coverage rather than fine detail and theorems with proofs are kept at a minimum. The material is not clouded by functional analytic and group theoretical definitions, and so is intelligible to readers with a general mathematical background. Some of the topics covered are scarcely covered elsewhere. Most of the material in Chapters 9-12 and 16-21 is at postgraduate level and has been influenced by the author's own research interests. There is more theory in these chapters than in the rest of the book since it is not easily accessed anywhere else. It has been found that these chapters are especially useful as reference material for senior undergraduate project work. The theory in other chapters of the book is dealt with more comprehensively in other texts, some of which may be found in the references section of the corresponding chapter. The book has a very hands-on approach and takes the reader from the basic theory right through to recently published research material. 
Python is extremely popular with a wide range of researchers from all sorts of disciplines; it has a very user-friendly interface and has extensive visualization and numerical computation capabilities. It is an ideal package to adopt for the study of nonlinear dynamical systems; the numerical algorithms work very quickly, and complex pictures can be plotted within seconds.

The first chapter provides an efficient tutorial introduction to Python. Simple Python programming is introduced using three basic programming structures: defining functions, for loops, and if, then, else constructs. New users will find the tutorials will enable them to become familiar with Python within a few days. Both engineering and mathematics students appreciate this method of teaching and I have found that it generally works well with one staff member to about twenty students in a computer laboratory. In most cases, I have chosen to list the Python commands at the end of each chapter; this avoids unnecessary cluttering in the text. The Python programs have been kept as simple as possible and should run under later versions of the package. All Python files for the book (including updates and extra files) can even be downloaded from the Web via GitHub at:

https://github.com/springer-math/dynamical-systems-with-applicationsusing-python

Readers will find that they can reproduce the figures given in the text, and then it is not too difficult to change parameters or equations to investigate other systems.

Chapters 2-12 deal with continuous dynamical systems. Chapters 2 and 3 cover some theory of ordinary differential equations and applications to models in the real world are given. The theory of differential equations applied to chemical kinetics and electric circuits is introduced in some detail. The memristor is introduced and one of the most remarkable stories in the history of mathematics is relayed. Chapter 2 ends with the existence and uniqueness theorem for the solutions of certain types of differential equations. The theory behind the construction of phase plane portraits for two-dimensional systems is dealt with in Chapter 3. Applications are taken from chemical kinetics, economics, electronics, epidemiology, mechanics, and population dynamics. The modeling of the populations of interacting species is discussed in some detail in Chapter 4 and domains of stability are discussed for the first time. Limit cycles, or isolated periodic solutions, are introduced in Chapter 5. Since we live in a periodic world, these are the most common type of solution found when modeling nonlinear dynamical systems. They appear extensively when modeling both the technological and natural sciences. Hamiltonian, or conservative, systems and stability are discussed in Chapter 6, and Chapter 7 is concerned with how planar systems vary depending upon a parameter. Bifurcation, bistability, multistability, and normal forms are discussed. 
The reader is first introduced to the concept of chaos in continuous systems in Chapters 8 and 9, where three-dimensional systems and Poincaré maps are investigated. These higher-dimensional systems can exhibit strange attractors and chaotic dynamics. One can rotate the three-dimensional objects in Python and plot time series plots to get a better understanding of the dynamics involved. Once again, the theory can be applied to chemical kinetics (including stiff systems), electric circuits, and epidemiology; a simplified model for the weather is also briefly discussed. Chapter 9 deals with Poincaré first return maps that can be used to untangle complicated interlacing trajectories in higher-dimensional spaces. A periodically driven nonlinear pendulum is also investigated by means of a nonautonomous differential equation. Both local and global bifurcations are investigated in Chapter 10. The main results and statement of the famous second part of David Hilbert's sixteenth problem are listed in Chapter 11. In order to understand these results, Poincaré compactification is introduced. The study of continuous systems ends with one of the authors specialities - limit cycles of Liénard systems. There is some detail on Liénard systems, in particular, in this part of the book, but they do have a ubiquity for systems in the plane. Chapter 12 provides an introduction to delay differential equations with applications in biology and nonlinear optics.

Chapters 13-17 deal with discrete dynamical systems. Chapter 13 starts with a general introduction to iteration and linear recurrence (or difference) equations. The bulk of the chapter is concerned with the Leslie model used to investigate the population of a single species split into different age classes. Harvesting and culling policies are then investigated and optimal solutions are sought. Nonlinear discrete dynamical systems are dealt with in Chapter 14 . Bifurcation diagrams, chaos, intermittency, Lyapunov exponents, periodicity, quasiperiodicity, and universality are some of the topics introduced. The theory is then applied to real-world problems from a broad range of disciplines including population dynamics, biology, economics, nonlinear optics, and neural networks. Chapter 15 is concerned with complex iterative maps in the Argand plane, where Julia sets and the now-famous Mandelbrot set are plotted. Basins of attraction are investigated for these complex systems and Newton fractals are introduced. As a simple introduction to optics, electromagnetic waves and Maxwell's equations are studied at the beginning of Chapter 16. Complex iterative equations are used to model the propagation of light waves through nonlinear optical fibers. A brief history of nonlinear bistable optical resonators is discussed and the simple fiber ring resonator is analyzed in particular. Chapter 16 is devoted to the study of these optical resonators, and there is discussion on phenomena such as bistability, chaotic attractors, feedback, hysteresis, instability, linear stability analysis, multistability, nonlinearity, and steady states. The first and second iterative methods are defined in this chapter. Some simple fractals may be constructed 
using pencil and paper in Chapter 17, and the concept of fractal dimension is introduced. Fractals may be thought of as identical motifs repeated on ever-reduced scales. Unfortunately, most of the fractals appearing in nature are not homogeneous but are more heterogeneous, hence the need for the multifractal theory given later in the chapter. It has been found that the distribution of stars and galaxies in our universe is multifractal, and there is even evidence of multifractals in rainfall, stock markets, and heartbeat rhythms. Applications in geoscience, materials science, microbiology, and image processing are briefly discussed. Chapter 18 provides a brief introduction to image processing which is being used more and more by a diverse range of scientific disciplines, especially medical imaging. The fast Fourier transform is introduced and has a wide range of applications throughout the realms of science.

Chapter 19 is devoted to the new and exciting theory behind chaos control and synchronization. For most systems, the maxim used by engineers in the past has been "stability good, chaos bad," but more and more nowadays this is being replaced with "stability good, chaos better." There are exciting and novel applications in cardiology, communications, engineering, laser technology, and space research, for example. A brief introduction to the enticing field of neural networks is presented in Chapter 20. Imagine trying to make a computer mimic the human brain. One could ask the question: In the future will it be possible for computers to think and even be conscious? The human brain will always be more powerful than traditional, sequential, logic-based digital computers and scientists are trying to incorporate some features of the brain into modern computing. Neural networks perform through learning and no underlying equations are required. Mathematicians and computer scientists are attempting to mimic the way neurons work together via synapses; indeed, a neural network can be thought of as a crude multidimensional model of the human brain. The expectations are high for future applications in a broad range of disciplines. Neural networks are already being used in machine learning and pattern recognition (computer vision, credit card fraud, prediction and forecasting, disease recognition, facial and speech recognition), the consumer home entertainment market, psychological profiling, predicting wave over-topping events, and control problems, for example. They also provide a parallel architecture allowing for very fast computational and response times. In recent years, the disciplines of neural networks and nonlinear dynamics have increasingly coalesced and a new branch of science called neurodynamics is emerging. Lyapunov functions can be used to determine the stability of certain types of neural network. There is also evidence of chaos, feedback, nonlinearity, periodicity, and chaos synchronization in the brain.

Chapter 21 focuses on binary oscillator computing, the subject of UK, International, and Taiwanese patents. The author and his co-inventor, Jon 
Borresen, came up with the idea when modeling connected biological neurons. Binary oscillator technology can be applied to the design of arithmetic logic units (ALUs), memory, and other basic computing components. It has the potential to provide revolutionary computational speed-up, energy saving, and novel applications and may be applicable to a variety of technological paradigms including biological neurons, complementary metal-oxidesemiconductor (CMOS), memristors, optical oscillators, and superconducting materials. The research has the potential for MMU and industrial partners to develop super fast, low-power computers and may provide an assay for neuronal degradation for brain malfunctions such as Alzheimer's, epilepsy, and Parkinson's disease!

Examples of coursework and three examination-type papers are listed in Chapter 22, and a complete set of solutions for the book is listed in Chapter 23.

Both textbooks and research papers are presented in the list of references. The textbooks can be used to gain more background material, and the research papers have been given to encourage further reading and independent study.

This book is informed by the research interests of the author, which are currently nonlinear ordinary differential equations, nonlinear optics, multifractals, neural networks, and binary oscillator computing. Some references include recently published research articles by the author along with two patents.

The prerequisites for studying dynamical systems using this book are undergraduate courses in linear algebra, real and complex analysis, calculus, and ordinary differential equations; a knowledge of a computer language such as Basic, C, or Fortran would be beneficial but not essential.

\section{Recommended Textbooks}

[1] H.P Langtangen and A. Logg, Solving PDEs in Python: The FEniCS Tutorial I (Simula SpringerBriefs on Computing), Springer, New York, 2017.

[2] B. Bhattacharya and M. Majumdar, Random Dynamical Systems in Finance, Chapman \& Hall/CRC, New York, 2016.

[3] L.C. de Barros, R.C. Bassanezi and W.A. Lodwick, A First Course in Fuzzy Logic, Fuzzy Dynamical Systems, and Biomathematics: Theory and Applications, Springer, New York, 2016.

[4] V. Volterra, Theory of Functionals and of Integral and Integro-Differential Equations, Dover Publications, New York, 2005. 
[5] J. Mallet-Paret (Editor), J. Wu (Editor), H. Zhu (Editor), Y. Yi (Editor), Infinite Dimensional Dynamical Systems (Fields Institute Communications), Springer, New York, 2013.

[6] C. Bernido, M.V. Carpio-Bernido, M. Grothaus et al., Stochastic and Infinite Dimensional Analysis, Birkhäuser, New York, 2016.

Special thanks go to Ben Nuttall (Python guru), Community Manager, the Raspberry Pi Foundation, Cambridge, UK (www.raspberrypi.org), for reviewing this book. I would also like to express my sincere thanks to all of the reviewers of this book and the other editions of my books. As always, thanks also go to Birkhäuser and Springer, especially Samuel DiBella (Assistant Editor, Springer Nature). Finally, thanks to my family and especially my wife Gaynor, and our children, Sebastian and Thalia, for their continuing love, inspiration, and support.

Manchester, UK

Stephen Lynch FIMA SFHEA 


\section{Contents}

1 A Tutorial Introduction to Python 1

1.1 The IDLE Integrated Development Environment for Python 2

1.1.1 Tutorial One: Using Python as a Powerful Calculator 4

1.1.2 Tutorial Two: Simple Programming with Python . . . 6

1.1.3 Tutorial Three: Simple Plotting Using the Turtle Module . . . . . . . . . . . . . . . 9 9

1.2 Anaconda, Spyder and the Libraries,

Sympy, Numpy, and Matplotlib . . . . . . . . . . . . . . . 14

1.2.1 Tutorial One: A Tutorial Introduction to Sympy . . . 15

1.2.2 Tutorial Two: A Tutorial Introduction to Numpy and Matplotlib . . . . . . . . . . . . . . 18

1.2.3 Tutorial Three: Simple Programming, Solving ODEs, and More Detailed Plots . . . . . . . . . . 20

1.3 Exercises . . . . . . . . . . . . . . . . 27

2 Differential Equations 33

2.1 Simple Differential Equations and Applications . . . . . . 34

2.1.1 Linear Differential Equations . . . . . . . . . . . . . 34

2.1.2 Separable Differential Equations _. . . . . . . . . . 35

2.1.3 Exact Differential Equations _. . . . . . . . . . . 39

2.1.4 Homogeneous Differential Equations . . . . . . . . . . 40

2.2 Applications to Chemical Kinetics . . . . . . . . . . . . . 44

2.3 Applications to Electric Circuits . . . . . . . . . . . . 48

2.4 Existence and Uniqueness Theorem . . . . . . . . . . . . . 53

2.5 Python Programs . . . . . . . . . . . . . . . 57

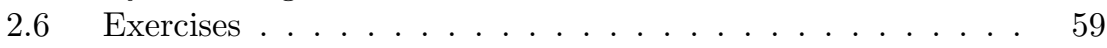

3 Planar Systems $\quad 65$

3.1 Canonical Forms . . . . . . . . . . . . . . . . . 66

3.1.1 Real Distinct Eigenvalues . . . . . . . . . . . . . . 68

3.1.2 Complex Eigenvalues $(\lambda=\alpha \pm i \beta) \ldots \ldots . \ldots 69$

3.1.3 Repeated Real Eigenvalues . . . . . . . . . . . 70 
3.2 Eigenvectors Defining Stable and Unstable Manifolds . . . . 72

3.3 Phase Portraits of Linear Systems in the Plane . . . . . . . 74

3.4 Linearization and Hartman's Theorem . . . . . . . . . . . 78

3.5 Constructing Phase Plane Diagrams . . . . . . . . . . . . . 79

3.6 Python Programs ................. . . 87

$3.7 \quad$ Exercises . . . . . . . . . . . . . . . 90

4 Interacting Species $\quad \mathbf{9 5}$

4.1 Competing Species . . . . . . . . . . . . . . . 96

4.2 Predator-Prey Models . . . . . . . . . . . . . . . . . 99

4.3 Other Characteristics Affecting Interacting Species . . . . . 104

4.4 Python Programs . . . . . . . . . . . . . . . . 107

4.5 Exercises ...................... 108

5 Limit Cycles $\quad \mathbf{1 1 3}$

5.1 Historical Background . . . . . . . . . . . . . . . . 114

5.2 Existence and Uniqueness of Limit Cycles in the Plane . . . 117

5.3 Nonexistence of Limit Cycles in the Plane . . . . . . . . . . 123

5.4 Perturbation Methods . . . . . . . . . . . . 127

5.5 Python Programs . . . . . . . . . . . . . . . . 136

5.6 Exercises ............................ 139

6 Hamiltonian Systems, Lyapunov Functions, and Stability $\mathbf{1 4 5}$

6.1 Hamiltonian Systems in the Plane . . . . . . . . . . . . . . . 146

6.2 Lyapunov Functions and Stability . . . . . . . . . . . . . 151

6.3 Python Programs ... . . . . . . . . . . . . 157

6.4 Exercises . . . . . . . . . . . . . . . . . 158

7 Bifurcation Theory $\quad 163$

7.1 Bifurcations of Nonlinear Systems in the Plane . . . . . . . . 164

7.1.1 A Saddle-Node Bifurcation . . . . . . . . . . . . 165

7.1.2 A Transcritical Bifurcation ... . . . . . . . . . 167

7.1.3 A Pitchfork Bifurcation . . . . . . . . . . . . . 167

7.1.4 A Hopf Bifurcation . . . . . . . . . . . . . . . . 170

7.2 Normal Forms . . . . . . . . . . . . . . . . . . . 170

7.3 Multistability and Bistability . . . . . . . . . . . . . 174

7.4 Python Programs . . . . . . . . . . . . . . . . 178

7.5 Exercises ..................... 180

8 Three-Dimensional Autonomous Systems and Chaos $\quad 185$

8.1 Linear Systems and Canonical Forms . . . . . . . . . . . . 186

8.2 Nonlinear Systems and Stability . . . . . . . . . . . . . . 190

8.3 The Rössler System and Chaos . . . . . . . . . . . . . . . . 194 
8.3.1 The Rössler Attractor . . . . . . . . . . . . . . . . . . 194

8.3 .2 Chaos . . . . . . . . . . . . . . 196

8.4 The Lorenz Equations, Chua's Circuit, and the

Belousov-Zhabotinski Reaction . . . . . . . . . . . . . . . . 199

8.4.1 The Lorenz Equations . . . . . . . . . . . . . . . . . . 199

8.4 .2 Chua's Circuit . . . . . . . . . . . . . . . 201

8.4.3 The Belousov-Zhabotinski (BZ) Reaction . . . . . . . 204

8.5 Python Programs . . . . . . . . . . . . . . . . 207

8.6 Exercises . . . . . . . . . . . . . . . 211

9 Poincaré Maps and Nonautonomous Systems in the Plane 215

9.1 Poincaré Maps . . . . . . . . . . . . . . . . . . 216

9.2 Hamiltonian Systems with Two Degrees of Freedom . . . . . 221

9.3 Nonautonomous Systems in the Plane . . . . . . . . . . . 227

9.4 Python Programs . . . . . . . . . . . . . . . . . . 235

$9.5 \quad$ Exercises . . . . . . . . . . . . . . . . . . 239

10 Local and Global Bifurcations 245

10.1 Small-Amplitude Limit Cycle

Bifurcations . . . . . . . . . . . . . . 246

10.2 Gröbner Bases . . . . . . . . . . . . . . . . . . . 252

10.3 Melnikov Integrals and Bifurcating Limit Cycles from a Center . . . . . . . . . . . . . . 258

10.4 Bifurcations Involving Homoclinic Loops . . . . . . . . . 260

10.5 Python Programs . . . . . . . . . . . . . . . 262

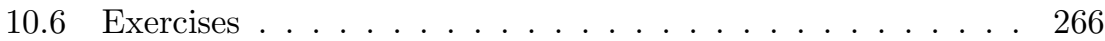

11 The Second Part of Hilbert's Sixteenth Problem 271

11.1 Statement of Problem and Main Results . . . . . . . . . . . 272

11.2 Poincaré Compactification . . . . . . . . . . . . . 275

11.3 Global Results for Liénard Systems . . . . . . . . . . . . . 281

11.4 Local Results for Liénard Systems . . . . . . . . . . . . . . 290

11.5 Python Programs . . . . . . . . . . . . . . . . . . . . . . 291

11.6 Exercises . . . . . . . . . . . . . . . . . . . . 292

12 Delay Differential Equations $\quad 297$

12.1 Introduction and the Method of Steps . . . . . . . . . . 298

12.2 Applications in Biology . . . . . . . . . . . . . . . . . . 304

12.3 Applications in Nonlinear Optics . . . . . . . . . . . 310

12.4 Other Applications . . . . . . . . . . . . . . . . . . . 313

12.5 Python Programs . . . . . . . . . . . . . . . . 315

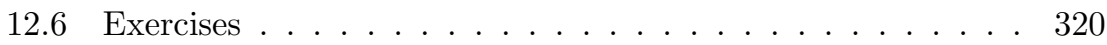


13 Linear Discrete Dynamical Systems $\quad \mathbf{3 2 7}$

13.1 Recurrence Relations . . . . . . . . . . . . . . . . . . . . . . 328

13.2 The Leslie Model . . . . . . . . . . . . . . . . . . . . 333

13.3 Harvesting and Culling Policies . . . . . . . . . . . . 337

13.4 Python Programs . . . . . . . . . . . . . . . . 342

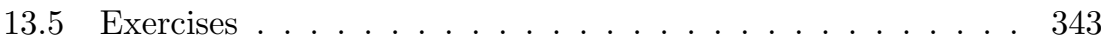

14 Nonlinear Discrete Dynamical Systems $\quad \mathbf{3 4 7}$

14.1 The Tent Map and Graphical Iterations . . . . . . . . . . . . 348

14.2 Fixed Points and Periodic Orbits . . . . . . . . . . . . . 353

14.3 The Logistic Map, Bifurcation Diagram, and Feigenbaum Number . . . . . . . . . . . . . . . 360

14.4 Gaussian and Hénon Maps . . . . . . . . . . . . . . . . . . . 368

14.5 Applications ..................... 373

14.6 Python Programs . . . . . . . . . . . . . . . . 376

14.7 Exercises ..................... 380

15 Complex Iterative Maps $\mathbf{3 8 5}$

15.1 Julia Sets and the Mandelbrot Set . . . . . . . . . . . . . . . 386

15.2 Boundaries of Periodic Orbits . . . . . . . . . . . . . . 391

15.3 The Newton Fractal . . . . . . . . . . . . . . . . . . . 395

15.4 Python Programs . . . . . . . . . . . . . . . 396

15.5 Exercises . . . . . . . . . . . . . . . . 399

16 Electromagnetic Waves and Optical Resonators $\quad 403$

16.1 Maxwell's Equations and Electromagnetic Waves . . . . . . 404

16.2 Historical Background . . . . . . . . . . . . . . . 406

16.3 The Nonlinear SFR Resonator . . . . . . . . . . . . . . . . 412

16.4 Chaotic Attractors and Bistability . . . . . . . . . . . . 413

16.5 Linear Stability Analysis . . . . . . . . . . . . . . . . . 417

16.6 Instabilities and Bistability . . . . . . . . . . . . . . . . . 420

16.7 Python Programs . . . . . . . . . . . . . . . . . . . . 424

16.8 Exercises . . . . . . . . . . . . . . . . . 428

17 Fractals and Multifractals $\quad \mathbf{4 3 3}$

17.1 Construction of Simple Examples . . . . . . . . . . . . . . . 434

17.2 Calculating Fractal Dimensions . . . . . . . . . . . . . . . . 441

17.3 A Multifractal Formalism . . . . . . . . . . . . . . . 448

17.4 Multifractals in the Real World and Some Simple Examples 452

17.5 Python Programs ... . . . . . . . . . . . . 459

17.6 Exercises . . . . . . . . . . . . . . . . 464 
18 Image Processing with Python $\quad 471$

18.1 Image Processing and Matrices . . . . . . . . . . . . . . . . 472

18.2 The Fast Fourier Transform . . . . . . . . . . . . . . . . . . 477

18.3 The Fast Fourier Transform on Images . . . . . . . . . . . . 484

18.4 Exercises . . . . . . . . . . . . . . . 487

19 Chaos Control and Synchronization $\quad 491$

19.1 Historical Background . . . . . . . . . . . . . . . . . . 492

19.2 Controlling Chaos in the Logistic Map . . . . . . . . . . . . 497

19.3 Controlling Chaos in the Hénon Map . . . . . . . . . . . . . 498

19.4 Chaos Synchronization . . . . . . . . . . . . . 505

19.5 Python Programs ................. 509

19.6 Exercises ................... 513

20 Neural Networks $\quad \mathbf{5 1 9}$

20.1 Introduction . . . . . . . . . . . . . . . . . . 520

20.2 The Delta Learning Rule and Backpropagation . . . . . . . 526

20.3 The Hopfield Network and Lyapunov Stability . . . . . . . . 531

20.4 Neurodynamics .................. . . 541

20.5 Python Programs . . . . . . . . . . . . . . . . . 545

20.6 Exercises . . . . . . . . . . . . . . . 550

21 Binary Oscillator Computing $\quad 557$

21.1 Brain Inspired Computing . . . . . . . . . . . . . . . . . 558

21.2 Oscillatory Threshold Logic . . . . . . . . . . . . . . . 563

21.3 Applications and Future Work . . . . . . . . . . . . . . 571

21.4 An Assay for Neuronal Degradation . . . . . . . . . . . . . . 577

21.5 Python Programs . . . . . . . . . . . . . . . . 579

21.6 Exercises . . . . . . . . . . . . . . . . . 584

22 Coursework and Examination-Type Questions $\quad 591$

22.1 Examples of Coursework Questions . . . . . . . . . . . 592

22.2 Examination 1. . . . . . . . . . . . . . . . 607

22.3 Examination 2 . . . . . . . . . . . . . . . . . 610

22.4 Examination 3 . . . . . . . . . . . . . . . 613

$\begin{array}{lr}23 \text { Solutions to Exercises } & \mathbf{6 1 9}\end{array}$

23.1 Chapter $1 \ldots \ldots . \ldots . \ldots . . \ldots 619$

23.2 Chapter 2 . . . . . . . . . . . . . . . . . . . . 622

23.3 Chapter 3 ....................... 623

23.4 Chapter 4 ..................... . . . 625

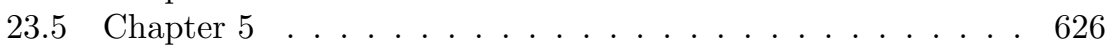

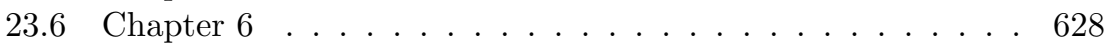

23.7 Chapter 7 . . . . . . . . . . . . . . . . . . . . . 628 


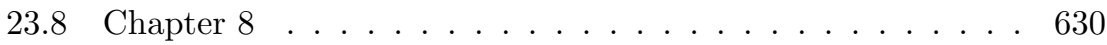

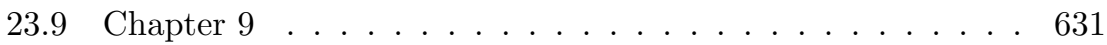

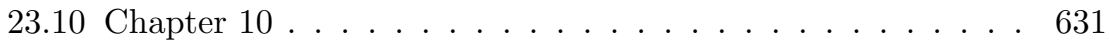

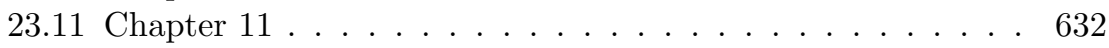

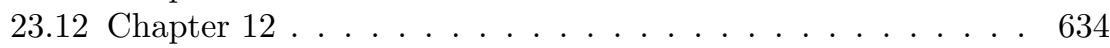

23.13 Chapter 13 .................... 634

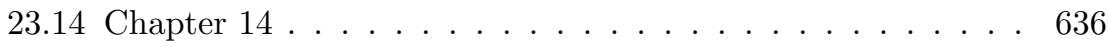

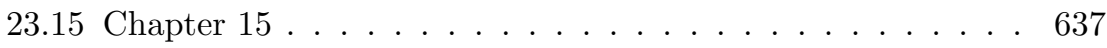

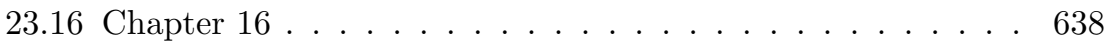

23.17 Chapter 17 . . . . . . . . . . . . . . . . . 638

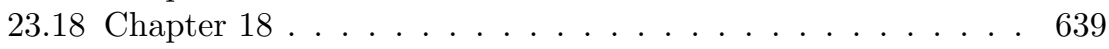

23.19 Chapter 19 . . . . . . . . . . . . . . . . . . 639

23.20 Chapter 20 . . . . . . . . . . . . . . . . . . 640

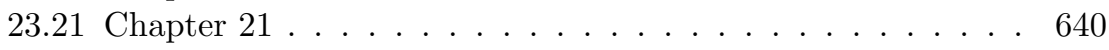

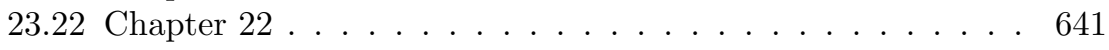

Appendix A Index of Python Programs $\quad \mathbf{6 4 5}$

A.1 IDLE Python Programs . . . . . . . . . . . . . . . . . . . 645

A.2 Anaconda Python Programs ... . . . . . . . . . . . 646

$\begin{array}{ll}\text { Index } & 651\end{array}$ 


\section{Appendix A}

\section{Index of Python Programs}

Readers can download the Python program files via GitHub:

https://github.com/springer-math/dynamical-systems-with-applicationsusing-python

These files will be kept up-to-date and extra files will be added in the forthcoming years.

\section{A.1 IDLE Python Programs}

These files include solutions to the Exercises listed in Chapter 1.

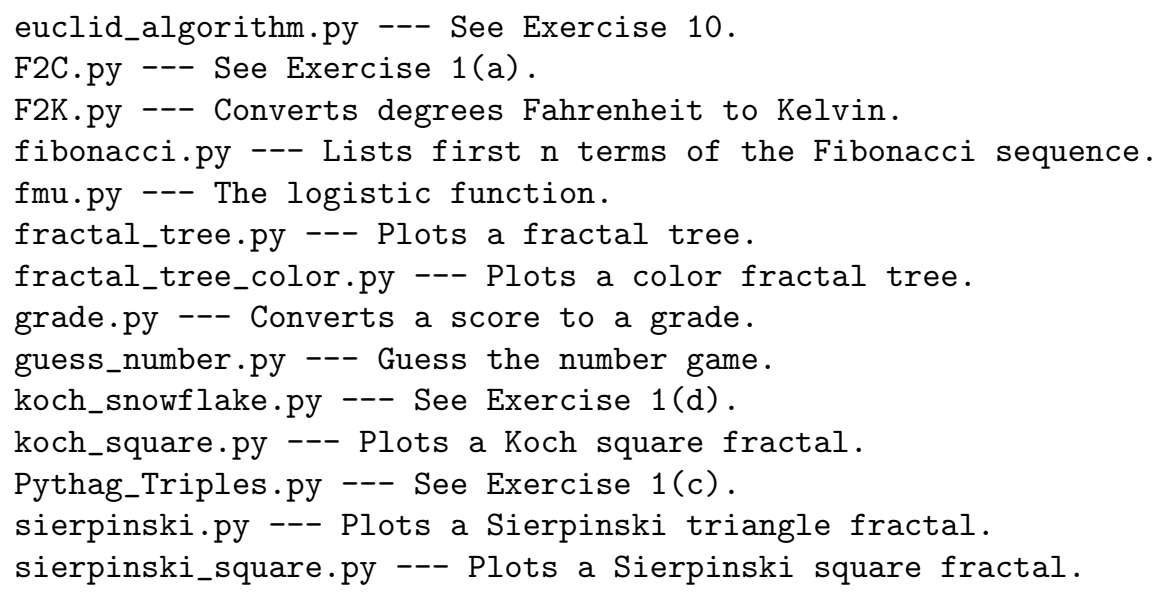


sum_primes.py --- See Exercise 1(b).

sum_n.py --- Sums the natural numbers to $n$.

\section{A.2 Anaconda Python Programs}

If you have difficulty with the animation programs in Spyder, you have to change the backend to run an animation in the IPython console. You can do that by running

\section{In [1]: \%matplotlib qt5}

before the animation. If you don't want to use this command every time, you can go to: Tools, Preferences, IPython Console, Graphics, Backend, and change it from "Inline" to "Automatic."

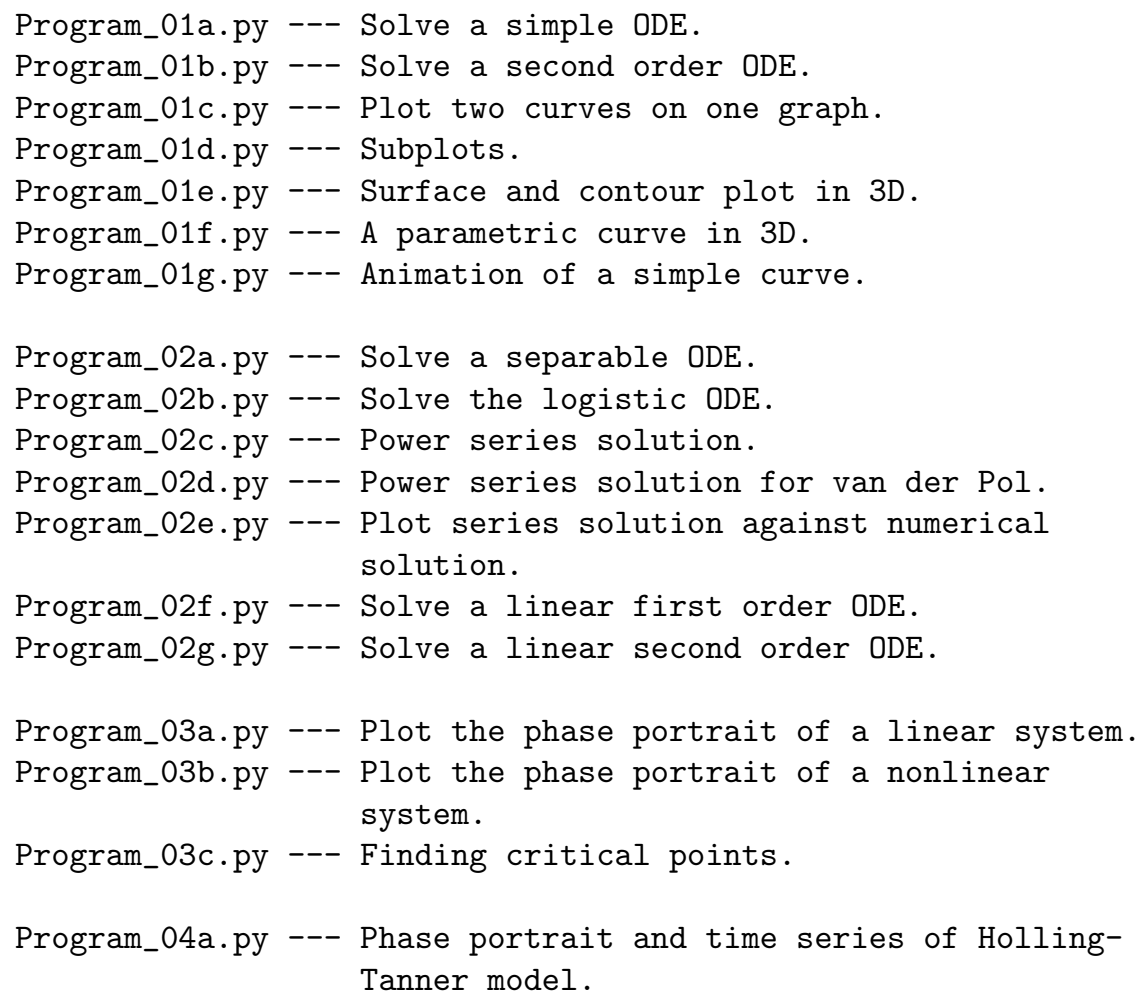




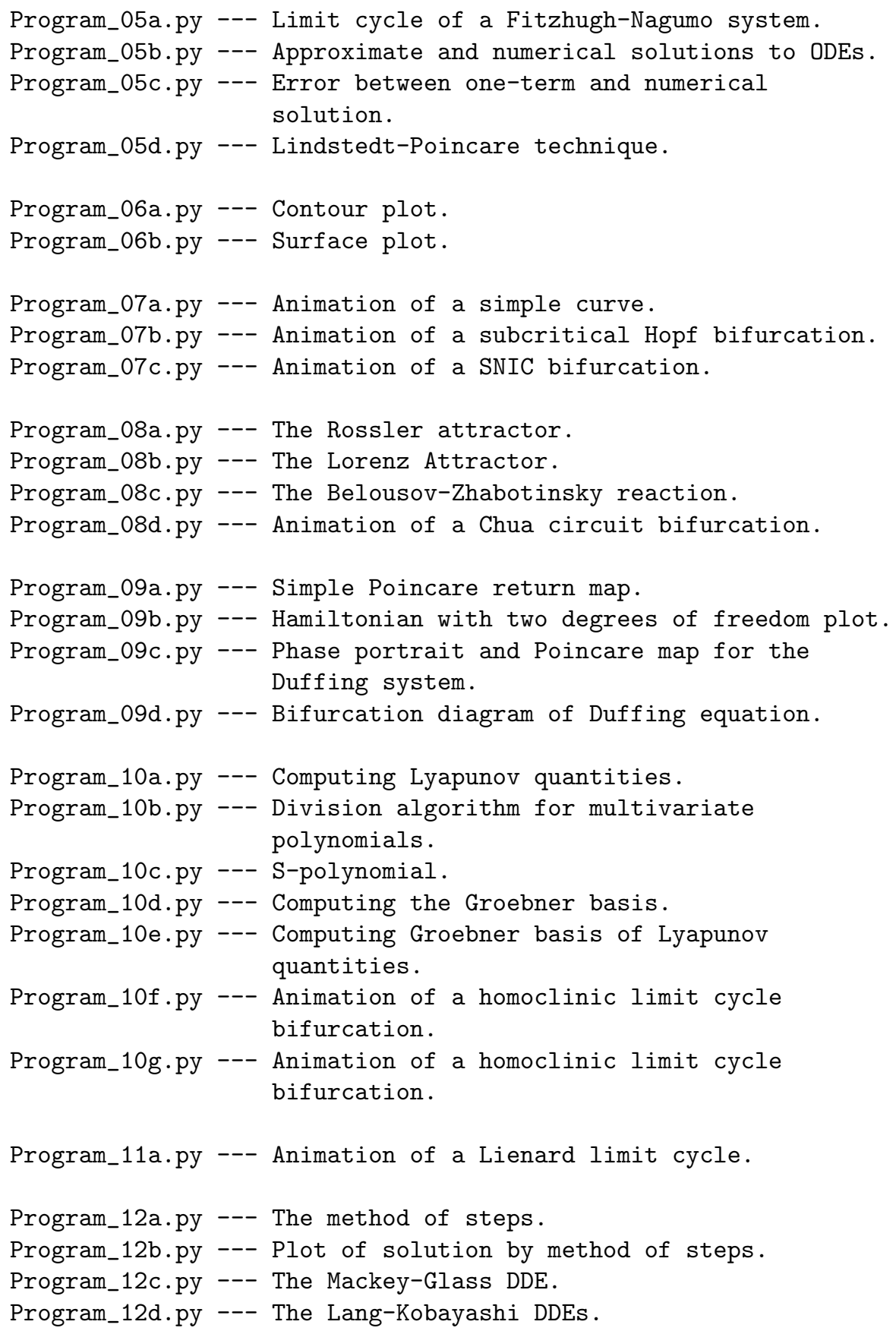




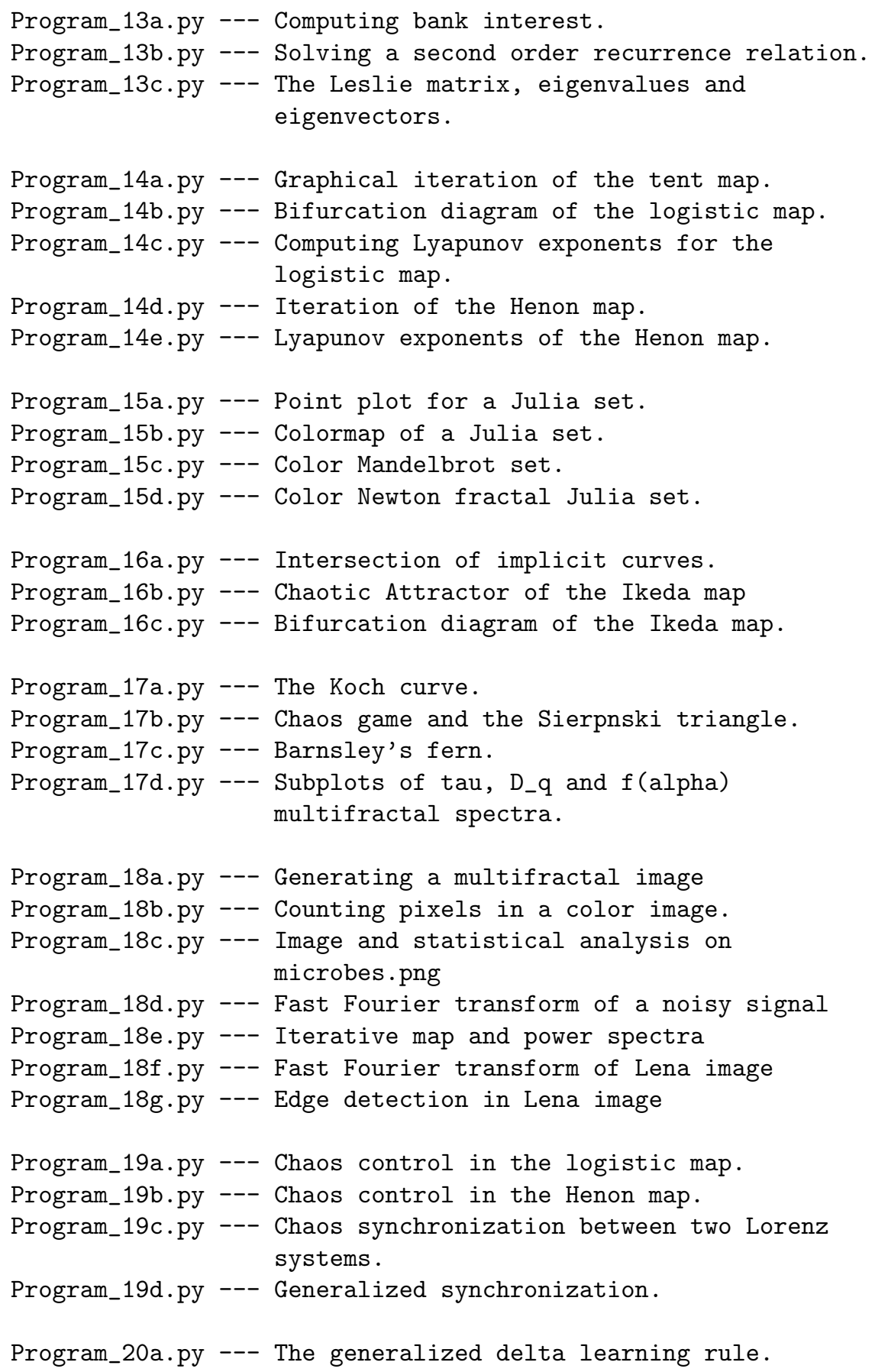


Program_20b.py --- The discrete Hopfield network.

Program_20c.py --- Iteration of a minimal chaotic neuromodule.

Program_20d.py --- Bifurcation diagram of neuromodule.

Program_21a.py --- The Hodgkin-Huxley equations.

Program_21b.py --- The Fitzhugh-Nagumo half-adder.

Program_21c.py --- Phase portrait Josephson junction limit cycle.

Program_21d.py --- Animated Josephson junction limit cycle.

Program_21e.py --- Pinched hysteresis of a memristor. 


\section{Index}

absorptive nonlinearity, 407

action potential, 114, 559

activation

function, 521, 550

level, 532

potential, 522

ADALINE network, 524

affine linear transformation, 440

age class, 106, 333

Airy equation, 61

algebraicity of limit cycles, 283

All or None principle, 560

Alzheimer's disease, 577

ampere, 48

Ampere's law, 405

Anaconda, 14

Anaconda Python programs, 646

anemia, 374

angiogenesis, 308

angular frequency of the wave, 406

animation, 26

Spyder, 646

ants and termites, 96

aperiodic, 201, 360

behavior, 196

append, 262

applying a damper, 493

arrhythmic, 495

Artificial Intelligence Group, 526

artificial neural networks, 520
ArtistAnimation, 178

assay for neuronal degradation, 577

associative memory, 524, 531

asymptotic expansion, 127

asymptotically stable

critical point, 152

asynchronous updating, 536

attractor, 193

attributes, 529

autocatalysis, 204

autonomous differential equation, 54

autonomous system, 186

auxiliary system approach, 507

average Lyapunov exponent, 366

ax.set title, 235

Axes3D(fig), 235

axial flow compressors, 175

axon, 521,558

Baby computer, 571

backpropagation, 526 algorithm, 528

backward training, 388

ballistic propagation, 571

bandwidth, 407

Barnsley's fern, 441

basin of attraction, 159, 193, 372, 388

basis, 253

(C) Springer International Publishing AG, part of Springer Nature 2018

S. Lynch, Dynamical Systems with Applications using Python, https://doi.org/10.1007/978-3-319-78145-7 
batch data, 530

Belousov-Zhabotinski reaction, 204, 212

Bendixson's criterion, 123

bias, 521

bifurcating limit cycles from a center, 258

bifurcation

curve, 167

diagram, 166

at infinity, 273

point, 362

value, 164

bifurcation diagram

CR resonator, 409

DDE

field components, 313

Mackey-Glass, 307

Duffing equation, 232

Gaussian map, 370

Ikeda map, 425

Josephson junction, 575

limit cycles, 177

logistic map, 365

neuromodule, 544

periodically forced pendulum, 233

SFR resonator, 422

SNIC, 178

binarize, 475

binary half adder, 565

biology, 374

bipolar activation function, 522

bistability, 174, 175, 368, 421

bistable, 175, 206, 370, 408

cycle, 203

device, 407, 421

neuromodule, 543

optical resonator, 495

region, 233, 241, 409, 414

solution, 176

bistable region, 544

blit, 178 blowflies, 360

bluegill sunfish, 104

Boston housing data, 529

boundaries of periodic orbits, 391

box-counting dimension, 443, 448

brain functions, 521

Briggs-Rauscher, 204

bursting, 310

butterfly effect, 200

BZ reaction, 204

canny edge detector, 477

canonical form, 66, 67, 186

Cantor

multifractal, 454

set, 235,435

capacitance, 49

capacitor, 49

cardioid, 393

cardiology, 492

carrying capacity, 38

cavity ring (CR) resonator, 408

cavity round-trip time, 408

cell body, 558

center, 69, 148, 246

manifold

theorem, 191

changing the system parameters, 493

chaologist, 492

chaos, 194, 196, 350, 353, 409

control, 541, 552

synchronization, 505

chaos control

OGY method, 493

periodic proportional pulses, 513

chaos game, 439

chaotic

attractor, 197, 229, 415

dynamics, 199

phenomena, 348 
chaotic attractor

Hénon map, 501

neuromodule, 542

Sierpiński, 440

Chapman cycle, 211

characteristic

equation, 302, 330

exponent, 283

multiplier, 219

charge density, 405

chemical

kinetics, 44, 86, 117, 204, 505,561

reaction, 59

signals, 521

substance, 61

chemical law of mass action, 44

Chua's circuit, 51, 117, 201, 493, 514

circle map, 221

circular frequency of light, 410

classical symmetry argument, 248

classification of critical points, 71

climate change, 314

clipping problem, 451

clockwise bistable cycle, 632

clockwise hysteresis, 241, 410

cluster, 524

cmap, 396

CMOS oscillators, 571

coarse Hölder exponent, 450

codimension-1 bifurcation, 182

codimension-2 bifurcation, 182

coexistence, 96

coexisting chaotic attractors, 372

col, 69

collect, 128

$\operatorname{comb}(\mathrm{k}, \mathrm{s}), 459$

common typing errors, 20

commutative ring, 252

competing species, 96, 110, 140

complete synchronization, 506 completely

integrable, 221

reduced, 254

complex eigenvalues, 69, 330

complex iterative equation, 417

complex(x,y), 396

compound interest, 329

computer algebra, 252

concentrations, 44

conditional Lyapunov exponents, 506

conductivity, 406

conformal mapping, 386

conservation of energy, 146

conservation of mass, 86

conservative, 146

contact rate, 212

content-addressable memory, 532

continuation lines, 20

continuous Hopfield model, 532

contour plot, 24

control curves, 499

control engineering, 526

control parameter, 495

control region, 495

controlling chaos

Hénon map, 498

logistic map, 497

conversational agents, 526

convex closed curve, 123

convoluted surfaces, 191

coordinates of an image, 474

core area of the fiber, 413

corollary to Poincaré-Bendixson

theorem, 120

correlation dimension, 450

coulomb, 48

counterclockwise hysteresis, 410

coupler, 412

critical point, 55, 72, 186, 191, 300

at infinity, 276 
culling, 106 policy, 337

current, 48 density, 405

cusp, 84

cylindrical polar coordinates, 193

damping, 116

damping coefficient, 282

dangerous bifurcation, 174

Daphnia dentifera, 104

dashed curve, 22

data, 545

data mining, 520

databases, 529

DDE, 298

dde23, 315

ddeint, 298

def, 6

defibrillator, 495

defraction, 61

degenerate critical point, 148 node, 70

degree, 273

degree lexicographical order, 253

delay differential equation, 298

deleted neighborhood, 121

delta learning rule, 524, 526

dendrites, 521, 558

depolarization, 559

depolarize, 566

derivative of the Poincaré map test, 220

desired vector, 527

deterministic chaos, 195, 492

deterministic system, 520

$D_{f}, 441$

dielectric, 406

difference equation, 328, 551

differential amplifier, 407

diffusion limited aggregates

(DLA), 453 dimension, 448

direction

field, 66

vector, 66

discrete Fourier transform, 479

discrete Hopfield model, 536

dispersive nonlinearity, 407

displacement function, 247

distributive laws, 252

divergence test, 248

domain of stability, 97, 193, 388

double-coupler fiber ring resonator, 410, 428

double Hopf bifurcation, 308, 322

double-scroll attractor, 203

double-well potential, 151

$D_{q}, 448$

driver system, 506, 507

dsolve, 57

Duffing

equation, 129, 227

system, 493, 611

Dulac's criterion, 122

Dulac's theorem, 273

$E_{C}, 191$

economic model, 117, 322, 332

economics, 92, 374, 382, 453, 505

edge detection

Roberts, 485

Sobel, 485

eig, 342

eigenvector, 72

El Niño, 313

electric

circuit, 48, 92, 117, 201, 532

displacement, 405

displacement vector, 405

field, 412, 417, 495

field strength, 405

flux density, 405

electromotive force (EMF), 49

elementary steps, 44 
elliptic integral, 259

EMF, 49

energy level, 227

enrichment of prey, 106

ENSO model, 313

Enthought Canopy, 14

environmental effects, 106

environmental model, 313

epidemic, 61, 85, 106, 117, 212

epilepsy, 577

epoch, 524

equilibrium point, 55

ergodicity, 366, 495

error backpropagation rule, 528

error function, 527

erythrocytes, 374

$E_{S}, 72,186,191$

$E_{U}, 72,186,191$

Euclidean dimension, 448

Euclid's algorithm, 29

exact, 39

exact differential equation, 39

excitatory, 521, 558

existence and uniqueness limit cycle, 117

existence theorem, 53

extinct, 96

Fabry-Perot interferometer, 407 resonator, 407

farad, 49

Faraday's law, 49 of induction, 404

fast Fourier transform, 480

feedback, 175, 407, 420

feedback mechanism, 543

feedforward single layer network, 523

Feigenbaum constant, 365

Ferranti Mark 1, 571

FFT, 480

fiber parameters, 423
Fibonacci sequence, 343

field, 253

figsize, 107

fine focus, 246

first integral, 146

first iterative method, 419, 422, 552

first return map, 216

first-order difference equation, 328

fish population, 38, 181, 345

Fitzhugh-Nagumo

equations, 116

oscillator, 114

system, 563

fixed point, 55, 355

period $\mathrm{m}, 221$

period N, 357

period one, $217,414,497$

period two, 498

fixed size box-counting algorithm, 451

fixed weight box-counting algorithm, 451

flow, 118

focal values, 247

fold bifurcation, 182

for loop, 6

forced system, 227

forward rate constant, 46

fossil dating, 59

Fourier spectrum, 478

Fourier transform, 477

fractal, 434, 441

attractor, 197, 441

dimension, 441

Cantor set, 442

Koch curve, 442

Koch square, 442

Sierpiński triangle, 442

geometry, 434

structure, 196, 201, 386

fragmentation ratios, 449 
$f(\alpha)$ spectrum, 448

FuncAnimation, 178, 207

Function, 57

function approximators, 526

fundamental memory, 536

fuzzy discs, 451

Gauss's law

electricity, 405

magnetism, 405

Gauss-Newton method, 528

Gaussian input pulse, 421

Gaussian map, 368

Gaussian pulse, 638

generalized delta rule, 528

generalized fractal dimensions, 448

generalized mixed Rayleigh

Liénard equations, 267

generalized synchronization, 507

gestation period, 304

GitHub, 2

global bifurcation, 260, 273

global warming, 314

globally asymptotically stable, 194, 211

glucose in blood, 60

Gröbner bases, 252

gradient, 66

gradient vector, 527

graphene nano-ribbon, 573

graphic, 120

graphical method, 351, 417

gray scale, 458,473

Green's theorem, 122

Gross National Product (GNP), 374

Guido van Rossum, 2

Hénon-Heiles Hamiltonian, 225

haematopoiesis, 306

Hamiltonian, 146, 611
Hamiltonian systems with two degrees of freedom, 221

handcrafted patterns, 541

hard bifurcation, 174

Hartman's theorem, 79

harvesting, 106, 181

policy, 337

Hausdorff dimension, 448

Hausdorff index, 441

Hausdorff-Besicovich dimension, 450

Heaviside function, 523

Hebb's learning law, 522

Hebb's postulate of learning, 536

help command, 20

Hénon map, 370, 445, 451, 609, 612

henry, 49

heteroclinic

bifurcation, 234

orbit, 120, 150, 234, 274

tangle, 234

heterogeneous, 448

hidden layer, 524, 528, 603

high pass filter, 484

Hilbert numbers, 273

Hints for programming, 20

history, 176

history function, DDEs, 298

Hodgkin-Huxley equations, 114, 560

Holling-Tanner model, 101, 139, 164

homoclinic

bifurcation, 200, 234, 261

loop, 260, 261, 267

orbit, 150, 234, 274

tangle, 234

homogeneous, 448

homogeneous differential

equation, 40 
Hopf

bifurcation, 170, 182, 303

singularity, 182

Hopfield network, 156, 531, 551, 610, 613

Hopfield neural network, 525

horseshoe dynamics, 235

host-parasite system, 104

human population, 85, 343

hyperbolic

attracting, 283

critical point, 79

fixed point, 220, 371

iterated function system, 440

repelling, 283

stable limit cycle, 220

unstable limit cycle, 220

hyperpolarize, 566

hyperpolarized, 559

hysteresis, 175, 371, 421

curves, 313

Josephen junction, 574

IDE, 14

ideal, 252

IDLE, 2

IDLE Python programs, 645

if, elif, else, 6

Ikeda

DDE, 310

map, 375, 414, 428, 513

im, 396

image analysis, 453

image compression, 434, 484

incident, 407

indentation level, 20

index, 125

inductance, 49

infected population, 105

infectives, 85

inflation unemployment model, 382

infodict, 107 information dimension, 450

inhibitory, 521, 558

initial value problem, 38

input vector, 521

insect population, 109, 345, 616

instability, 421

instant physician, 526

integrable, 223

integrate and fire neuron, 116

Integrated Development

Environment, 2, 14

integrating factor, 34

intensity, 412

interacting species, 95, 626

intermittency, 203, 212, 363 route to chaos, 365

invariant, 118, 201, 416 axes, 82,98

inverse discrete Fourier transform, 479

inverted Koch snowflake, 609

inverted Koch square, 438

inverted pendulum, 322

io.imsave, 474

isoclines, 67

isolated periodic solution, 114

isothermal chemical reaction, 86

iterated function system (IFS), 440

iteration, 328

Jacobian, 171

Jacobian matrix, 78, 192, 205, 371,502

Jaynes-Cummings model, 595

Jordan curve, 121, 286

Josephson junction mathematical model, 573

Josephson junction (JJ), 571

jth point of period i, 357

Julia set, 386, 389, 434, 612 color, 390 
KAM

theorem, 227

tori, 227

kernel machines, 525

Kerr

effect, 407, 413

type, 413

kinetic energy, 146

Kirchhoff's

current law, 49

laws, 532

voltage law, 49

Koch

curve, 436

snowflake, 464

square, 436

ladybirds and aphids, 99

lambdas, 315

laminarize, 494

Lang-Kobayashi equations, 310

Laplace transform, 50

large-amplitude limit cycle, 175

bifurcation, 175

laser, 182, 374, 410, 494

model, 310

LaTeX, 24

law of mass action, 86

learning process, 521

learning rate, 527

least mean squared (LMS)

algorithm, 524

legend, 23, 107

Legendre transformation, 450

Leslie

matrix, 334

model, 333

lexicographical order, 253

lie detector, 526

Liénard

equation, 248

plane, 282 system, 116, 123, 139, 260, 281

large parameter, 286

local results, 290

theorem, 293

limit cycle, 106, 114, 118, 206, 595,611

hyperbolic, 259

neuron, 116

nonexistence, 608

3-D, 195

Lindstedt-Poincaré technique, 131 linear differential equation, 34

linear phase shift, 412, 423

linear stability analysis, 56, 300, 417

linear transformation, 187

linearization, 78

linearized system, 78

Lipschitz

condition, 53

continuous, 53

local bifurcation, 273

log-log plot, 445

logic gates, 407

logic operations, 565

logistic

equation, 38, 302

function, 6

growth, 101

map, 360, 497, 612

Lorenz

attractor, 201

equations, 199, 494

loss in the fiber, 412

Lotka-Volterra model, 99, 164, 212,304

low-gain saturation function, 523

low pass filter, 484

lowest common multiple, 256

Lyapunov

quantity, 247

stability, 531 
Lyapunov domain of stability, 155

Lyapunov exponent, 197, 366, 612

Lorenz system, 603

Lyapunov function, 151, 154, 194, 247, 284, 550, 607

Hopfield network, 532

Lyapunov quantities, 290

Lyapunov stability theorem, 152

lynx and snowshoe hares, 99

Mac OS, 2

Mackey-Glass model, 306

magnetic field vector, 405

magnetic flux, 405

magnetostrictive ribbon, 494

Mandelbrot, 443

Mandelbrot set, 389, 391, 434

manifold, 72

Maple, 26

math module, 4

Mathematica, 26

MATLAB, 26

MATLAB code to Python, 603

matplotlib, 18

maximal interval of existence, 54 , 62,118

Maxwell's equations, 404

Maxwell-Bloch equations, 406

Maxwell-Debye equations, 406

McCulloch-Pitts neuron, 522

MEA, 577

mean, 545

infectivity period, 212

latency period, 212

mechanical oscillator, 91

DDE, 321

mechanical system, 117, 176

Melnikov

function, 259

integral, 258

memory devices, 407

memristance, 52

memristor, 51, 572, 573 mathematical model, 574

meshgrid, 157

meteorology, 199

method of multiple scales, 134

method of steepest descent, 527

method of steps, 299

mgrid, 425

micro-parasite - zooplanktonfish system, 104

minimal chaotic neuromodule, 542

minimal Gröbner basis, 257

mixed fundamental memories, 537

mixing, 353

modulo, 254

monomial, 253

ordering, 253

mortgage assessment, 526

motif, 434

mplot3d, 207

multi-electrode array, 577

multidegree, 254

multifractal, 447, 472, 602

formalism, 448

Hénon map, 459

Sierpiński triangle, 459

spectra, 448

multistability, 174

multistable, 151, 175, 206, 241, 543

murder, 60

muscle model, 60

mutual exclusion, 96

myimages, 292

mylein sheath, 558

national income, 332

negative

limit set, 118

semiorbit, 118

negatively, invariant, 118

net reproduction rate, 339 
network architecture, 521

neural network, 375, 505, 521

DDE, 315

neuristor, 572

neurodynamics, 541

neurological assay, 577

neuromodule, 541

neuron

module, 375

neuron(s), 114, 521, 551, 558

neuronal model, 521

neurotransmitters, 558

Newton fractal, 395, 602

Newton's law of cooling, 60

Newton's law of motion, 146

Newton's method, 395, 528

noise, 497

NOLM, 404

with feedback, 410

nonautonomous system, 116, 227

nonconvex closed curve, 124

nondegenerate

critical point, 148, 246

nondeterministic chaos, 195, 492

nondeterministic system, 520

nonexistence of limit cycles, 123

nonhyperbolic

critical point, 79, 151, 607

fixed point, 371

nonlinear

center, 247

optics, 374

phase shift, 412

refractive index coefficient, 413

nonlinearity, 175, 407

nonperiodic behavior, 196

nonsimple canonical system, 67

normal form, 164, 170

normalized eigenvector, 340

not robust, 101

notebook, 14

np.mgrid, 88 nullclines, 67

numerical solutions, 42

numpy, 18

occasional proportional feedback (OPF), 494

ODE, 34

odeint, 42,57

OGY method, 495

ohm, 49

Ohm's law, 48

optical

bistability, 407

computer, 407

fiber, 410

fiber double ring, 410

memories, 407

oscillators, 572

resonator, 176

sensor, 408

optimal sustainable, 340

optogenetics, 578

orbit, 66,118

ordinary differential equation, 34

oscillation of a violin string, 114

oscillatory threshold logic, 563

output vector, 521

ozone production, 211

parasitic infection, 111

Parkinson's disease, 577

partial differential equations, 34

partition function, 448

Pascal's triangle, 464

passive circuit, 51

Peixoto's theorem in the plane, 164

pendulum, 147, 159, 241

double, 593

perceptron, 522

perihelion, 593

period, 259

bubblings, 368

limit cycle, 103, 119, 205 
undoublings, 368

period-doubling, 203

period-doubling bifurcations to chaos, 363

period-n cycle, 195

period-one behavior, 350

period-two, 196

behavior, 350

period-three behavior, 351

periodic

behavior, 115

orbit, 259

windows, 363

periodicity, 348, 353

permittivity of free space, 405

perturbation methods, 127

phase portrait, 66

phase shift, 412

physiology, 505

piecewise, 300, 315

piecewise linear function, 523

pinched hysteresis, 52, 574

pitchfork bifurcation, 167

pixels, 451

planar manifold, 187

plastics, 453

plt.axes, 292

Poincaré

compactification, 275

map, 119, 199, 216, 259, 371, 495

section, 216, 611

Poincaré-Bendixson theorem, 120, $227,281,287$

Poisson brackets, 223

polar coordinates, 69, 276

pole placement technique, 496

pollution, 106

polymer, 453

population, 92

of rabbits, 86

population model, 614 positive

limit set, 118

semiorbit, 118

positively, invariant, 118

potato man, 394

potential difference, 48

potential energy, 146, 151

potential function, 151

pow $(\mathrm{x}, \mathrm{y}), 315$

power, 412

law, 443

spectra, 199, 203

of a waterwheel, 92

power-splitting ratio, 412

pprint, 57

Prandtl number, 200

preallocate, 20

predation, 104 rate, 101

predator-prey, 117

DDE, 304

models, 99

system, 109

probe vector, 536

propagation, 412

psychological profiling, 526

PyDDE, 298

pydelay, 298, 311

Pyragas's method, 493

Python

based exam, 607, 613

files download, 2

qth moment, 448

qualitative behavior, 66

qualitatively equivalent, 74

quasi-periodicity, 221

quasi-polynomials, 302

quasiperiodic, 544, 552

route to chaos, 203

quasiperiodic forcing, 228

quiver, 88 
Rössler

attractor, 194

system, 194

radioactive decay, 610

randint(a,b), 459

random behavior, 195

Raspberry Pi, 2

rate constant, 45

rate-determining step, 45

Rational(1,2), 376

rationally independent, 221

ravel, 157

Rayleigh number, 200

Rayleigh system, 115

re, 396

reaction rate equation, 45

real distinct eigenvalues, 68

recurrence relation, 328

recurrent neural network, 524, 531

red blood cells, 374

red and grey squirrels, 96

reduced, 262

reduced Gröbner basis, 257

reflected, 407

refractive index, 407

refractive nonlinearity, 407

refuge, 106

regionprops, 477

regulator poles, 496

relative permeabilities, 406

relative permittivities, 406

repeated real eigenvalues, 70

repolarization, 559

resistance, 49

resonance terms, 173

resonant, 173

response system, 506, 508

restoring coefficient, 282

restoring force, 116

restrictions in programming, 291

return map, 247, 607

reverse rate constant, 46 reversed fundamental memories, 537

RGB image, 474

rgb2gray, 477

ring, 252

ringing, 241

RLC circuit, 51, 117

roach:fish population, 338

robust, 103

Rotating Wave Approximation

(RWA), 312

rsolve, 342

rubbers, 453

S-polynomial, 256

saddle point, 69, 148

saddle-node bifurcation, 165

saddle-node on an invariant cycle bifurcation, 176

safe bifurcation, 174

save image, 472

savefig, 22

scaling, 443, 448

scatter, 425

sea lions and penguins, 96

seasonal effects, 106

seasonality, 212

second iterative method, 420, 422,552

second order linear difference equation, 330

second part of Hilbert's sixteenth problem, 272

second-order differential equation, 50

secular term, 131

sedimentary rocks, 453

self-similar, 448

self-similar fractal, 441

self-similarity, 434

semistable

critical point, 56

limit cycle, 118, 220, 286 
sensitivity to initial conditions, 196, 348, 353, 492

separable differential equation, 35 separation of variables, 35

separatrix, 151

cycle, 261

series solutions, 42

SFR, 403

resonator, 409, 412

sharks and fish, 99

Sierpiński triangle, 439

sigmoid function, 523

signal processing, 453, 482

simple canonical system, 68

simple nonlinear pendulum, 146

simply connected domain, 123

singlet, 212

singular node, 70

Smale horseshoe map, 234, 373

Smale-Birkhoff theorem, 235

small perturbation, 56, 417

small-amplitude limit cycle, 246

soft bifurcation, 174

solar system, 492

solution curves, 36

solve, 88,258

soma, 521, 558

spatial vector, 405

spectrum of Lyapunov exponents, 197

speed of light, 406

spike train, 559

spin-glass states, 537

spirals, 355

spurious steady state, 537

SR flip-flop, 569

stability, 151, 190

diagram, 420

stable

critical point, 55, 152

fixed point, 361, 388

focus, 69

limit cycle, 103, 118 manifold, 72, 79, 186, 191, 495

node, 69

staircases, 356

stationary point, 55

std, 545

steady state, 51, 103

stem cell, 578

stiff system, 47, 212

stiffness, 116

stochastic methods, 520

stock market analysis, 453

stoichiometric equations, 45

Stokes's theorem, 405

strange attractor, 197

stretching and folding, 348

strictly dominant, 336

structurally

stable, 103, 164

unstable, 101, 164

subcritical Hopf bifurcation, 174, 200

subharmonic oscillations, 229

subplots, 22, 107

summing junction, 521

superconductor, 573

supercritical Hopf bifurcation, 174

supervised learning, 524

surface plot, 24

susceptible population, 105

susceptibles, 85

sustainable, 338

switches, 407

symbols, 57

sympy, 15

synaptic

cleft, 558

gap, 558

vesicles, 558

weights, 521

synchronization, 494, 505, 541 
synchronization of chaos, 505

synchronous updating, 537

target vector, 524, 527

targeting, 497

$\tau(q), 449$

Taylor series expansion, 56, 78, 371,417

tent map, 348, 608

$3 \mathrm{D}$ plot, 23

three-dimensional system, 186

threshold, 559

logic, 564

value, 85

time series, 105, 364

chaos detection, 198

plot, 198

Tinkerbell map, 601

Toda Hamiltonian, 240

topological dimension, 448

topologically equivalent, 74

torus, 228

total degree, 253

totally

connected, 388

disconnected, 388

training, 524

trajectory, 66, 118

transcritical bifurcation, 167

transfer function, 521, 551

transient, 51

transmitted, 407

transversal, 247

transversely, 216

travelling salesman problem, 531

triangular pulse, 421

trigsimp, 130

trivial fixed point, 356

turbulence, 453, 492

2D plot, 21

two-neuron module, 533 uint8, 476

unconstrained optimization

problem, 527

uncoupled, 187

uniform asymptotic expansion, 127

uniform harvesting, 341

unipolar activation function, 522

uniqueness theorem, 53

universality, 365

Unix, 2

unstable

critical point, 55, 153

fixed point, 361,388

focus, 69

limit cycle, 118

manifold, 72, 79, 186, 191

node, 68

unsupervised learning, 524

vacuum, 405

vacuum tube oscillator, 571

value of homes in Boston, 529

van der Pol equation, 130

van der Pol system, 114, 259

vector field, 66

plot, 533

velocity of light, 413

Verhulst's equation, 38, 96

virus, mobile phone, 592

viscosity, 200

viscous fingering, 434

volt, 48

voltage drop, 48

wave equations, 404

wave vector, 406

wavelength, 406

light, 413

$W_{C}, 191$

while loop, 6, 29 
Windows, 2

wing rock, 175

WinPython, 14

$W_{S}, 79,191$

$W_{U}, 79,191$

X-ray spectroscopy, 453
XOR gate, 522

You Tube, 386

youngest class harvesting, 339

$Z_{q}, 448$ 